> Maîtriser l'oxydation est indispensable pour gérer l'évolution des systèmes biologiques dans leur complexité, en particulier dans le cas des aliments dont la dégradation peut avoir des conséquences en sécurité alimentaire. Les différentes méthodes d'évaluation de l'activité antioxydante d'un additif alimentaire sont présentées après examen du contexte et des mécanismes d'oxydation. L'activité anti-oxydante est évaluée soit par le dosage des produits formés (en particulier des hydroperoxydes) par des techniques photométriques plus ou moins directes, soit par la mesure de l'efficacité du composé à piéger des radicaux libres. Les méthodes comparant le piégeage d'un additif avec celui d'un anti-oxydant de référence, le Trolox ${ }^{\circledR}$, sont applicables à des produits variés quelle que soit l'hydrophilie ou l'hydrophobie du milieu. <

\section{Méthodes d'évaluation du potentiel antioxydant dans les aliments}

\author{
Françoise Marc, André Davin, \\ Laurence Deglène-Benbrahim, Carine Ferrand, \\ Michel Baccaunaud, Pierre Fritsch
}

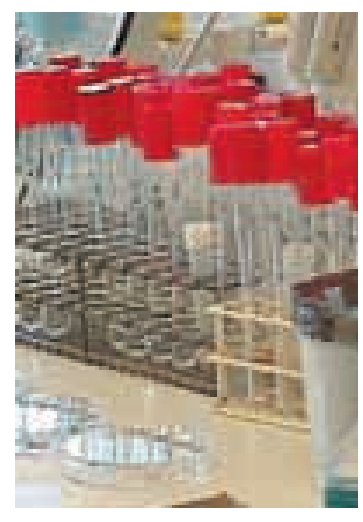

ignorée, il peut en découler de graves problèmes toxicologiques dus aux synergies ou aux produits dérivés. Ce constat pose le problème de l'évaluation de la présence des additifs en fonction des constituants de l'aliment. Leur utilisation dans les formulations doit apporter une valeur ajoutée aux aliments sans être préjudiciable à la santé humaine. Dans cette optique, un examen des techniques de dosage appropriées est envisagé afin d'évaluer l'efficacité des antioxydants et de limiter les ajouts aux doses strictement indispensables aux objectifs de préservation à atteindre. Cette étude bibliographique retient des méthodes directes ou indirectes, adaptées ou adaptables à l'évaluation du potentiel antioxydant des additifs. 


\section{Teneur en eau, stabilité des produits biologiques et potentiel redox}

Un équilibre permanent s'établit entre l'hygrométrie de l'atmosphère et l'eau présente dans le produit. Les composés solubles (sels, sucres, alcools, groupes hydrophiles...) mobilisent l'eau par solvatation. Si la teneur en eau atmosphérique croît, l'adsorption de l'eau se fait par couches successives de degré de liberté croissant avec leur éloignement du support. Les $\mathrm{a}_{\mathrm{w}}$ (rapport des quantités d'eau libre et totale) tant dans le produit que dans l'atmosphère en équilibre sont égales pour une température $\theta$ donnée, d'où :

$$
a_{w}=\frac{\text { pression partielle de l'eau dans l'aliment à } \theta^{\circ}}{\text { pression partielle de l'eau pure à } \theta^{\circ}}
$$

soit : $a_{w}$ (toujours comprise entre 0 et 1$)=(\%)$ humidité relative/ 100 .
La réactivité d'un produit est lié à son $\mathrm{a}_{\mathrm{w}}$. Si l'eau libre est nécessaire pour l'activité biologique, elle inhibe les réactions de composés à caractère hydrophobe strict (lipides), ou la réaction de Maillard ${ }^{1}$. La Figure 1 présente de façon synthétique les diverses évolutions responsables de dégradations en fonction de l'activité de l'eau $a_{w}[1]$. L'oxydation a lieu avec ou sans la participation de l'oxygène moléculaire ; elle se définit comme une perte d'électrons, le gain antagoniste correspondant à la réduction. D'après ce concept, un potentiel redox $\left(\varepsilon_{\text {ox/red }}^{\circ}\right)$ peut être affecté à chaque couple oxydant/réducteur impliqué, le couple possédant le potentiel élevé pouvant théoriquement oxyder ceux ayant des potentiels plus faibles. L' $\varepsilon^{\circ}{ }_{0 x / r e d}$ d'un système se mesure par rapport au potentiel électrochimique de l'électrode à hydrogène, fixé par convention à 0,0 volt à $\mathrm{pH}=0$.

Tant lors du stockage, qu'au cours des processus de transformation, le maintien d'une $a_{w}$ intermédiaire satisfaisante $(0,2-0,3)$ ne peut être garanti.

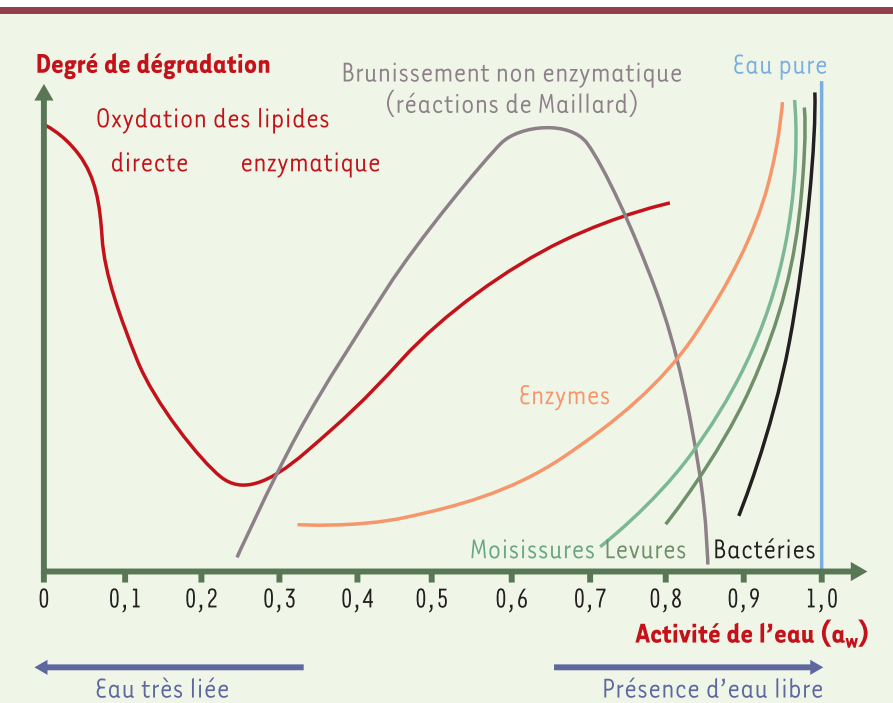

Figure 1. Risques de détérioration des aliments en fonction de l'activité de l'eau. L'activité de l'eau $\left(a_{w}\right)$ correspond au rapport entre la pression de vapeur d'eau de l'aliment et la pression de vapeur de l'eau pure à la même température. La valeur de l'a $a_{w}$ varie de 0 (produit sec au point que toute l'eau est liée à l'aliment, et donc sans qualité réactive) à $l$ (toute l'eau est libre). À une $a_{w}$ de 1 correspond de l'eau pure sans soluté difficile à atteindre et surtout à maintenir (ordonnée de droite en bleu). La courbe en rouge représente les risques oxydatifs : quand l'eau est très liée, l'oxydation est directe ; à partir d'un certain degré de liberté de l'eau, l'oxydation devient enzymatique (que les enzymes soient endogènes ou exogènes, provenant de micro-organismes ou du milieu). La courbe en gris correspond aux réactions de Maillard, non enzymatiques, qui s'opposent pour les humidités intermédiaires au brunissement enzymatique correspondant principalement à une oxydation des polyphénols. Les courbes en vert clair, vert foncé et noir correspondent au domaine d'influence de la bactériologie (respectivement moisissures, levures et bactéries) qui est limité à la présence d'eau libre (d'après [1]).

\section{Rappel sur les processus d'oxydation: conséquences et contrôle}

Les mécanismes d'oxydations des composés insaturés biologiques (acides gras, caroténoïdes, polyphénols...) sont souvent des réactions radicalaires avec l'oxygène moléculaire [2-4] et présentent trois phases principales. - La phase de déclenchement où se forme un premier radical libre. L'arrachement du proton est facilité tant par la chaleur (agitation moléculaire) que les rayonnements ou les catalyseurs (métaux tels que $\mathrm{Cu}, \mathrm{Fe}, \mathrm{Co}, \mathrm{Mn}, \mathrm{Ni}$...).

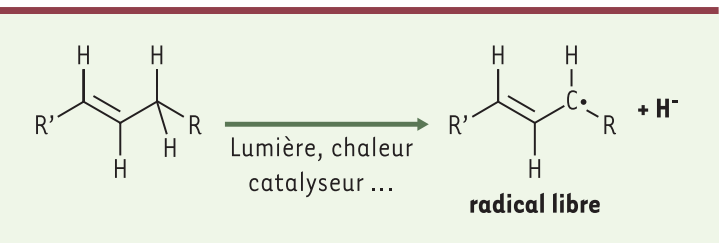

- La phase de propagation où l'oxygène fixé donne un radical peroxyle qui réagit avec une autre molécule et conduit à un néoradical libre et un hydroperoxyde.

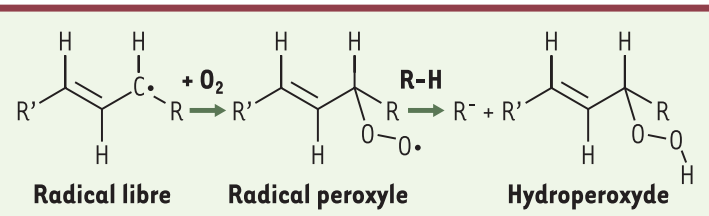

Les hydroperoxydes instables se scindent en composés plus courts [2].

${ }^{1}$ Les réactions de Maillard sont un ensemble complexe de réactions mettant en œuvre, dans des substrats biologiques ou agro-alimentaires, des composés présentant des groupements réducteurs et des composés aminés qui réagissent entre eux pour produire des substances plus ou moins aromatiques et colorées. 
- La phase de terminaison, où se recombinent différents radicaux formés.

Globalement, ce processus conduit à des hydrocarbures, des aldéhydes, des cétones, des acides, des esters, des peracides, des peroxydes, mais aussi à des produits de polymérisation. Sous son effet, l'aliment accuse une perte de qualité nutritionnelle ou organoleptique (rancissement, changement de couleur...).

Pour limiter l'oxydation, l'industrie agroalimentaire peut baisser le taux d'oxygène (immersion, vide, atmosphère sous azote), ralentir les réactions par réfrigération ou congélation, détruire les enzymes d'oxydation (polyphénol oxydases) par blanchiment, et user d'antioxydants inhibant l'oxydation induite par l'oxygène moléculaire.

En limitant les risques de radicaux libres, la présence d'antioxydants, combinée à d'autres techniques, est indispensable à la stabilité des produits. Si l'oxydation résulte uniquement d'une réaction avec l'oxygène, l'antioxydant est alors un antioxygène.

\section{Rôle et principaux types d'antioxydants alimentaires}

L'antioxydant alimentaire idéal, et facilement incorporable et efficace à faible dose, est non toxique, n'entraîne ni coloration, ni odeur, ni saveur indésirable. Résistant aux processus technologiques, il est stable dans le produit fini.

Les molécules à bas $\varepsilon^{\circ}{ }_{\text {ox/red }}$ aptes à ralentir, retarder ou prévenir les processus d'oxydation [5] sont en fait des agents de prévention ou de terminaison capables d'éviter ou de piéger les radicaux libres. L'action préventive bloque l'initiation en complexant les catalyseurs, en réagissant avec l'oxygène ou en déviant de l'aliment des effets de lumière ou des rayonnements.

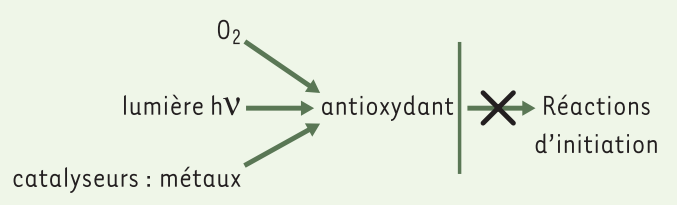

En tant qu'agents de terminaison (piégeage de radicaux), les antioxydants transforment les radicaux en composés plus stables et bloquent la phase de propagation. Un tel effet résulte d'une structure de donneurs de $\mathrm{H}^{\bullet}$ souvent aromatique, cas des dérivés du phénol (tocophérols, polyphénols, flavonoïdes...).

De nombreux polyphénols d'origine végétale : vitamine $\varepsilon$ (d-tocophérol), flavonoïdes et flavones, caroténoïdes et vitamine C (acide L-ascorbique) sont des antioxy-

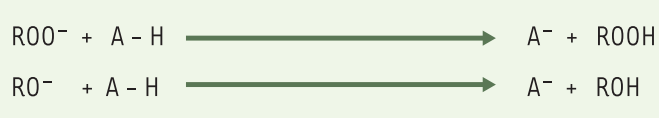

A - $\mathrm{H}$ : antioxydant, donneur de $\mathrm{H}^{-}$

dants naturels disponibles (Figure 2) [6]. Certains d'entre eux ( $\beta$-carotène, lycopène...) sont utilisés comme colorants.

D'autres composés phénoliques de synthèse comme le BHT (3,5-ditertiobutyl-4-hydroxytoluène), le BHA (3-tertiobutyl-4-hydroxyanisole), le TBHQ (tertiobutylhydroxyquinone) et le PG (gallate de propyle), sont autorisés dans certains aliments.

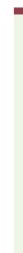

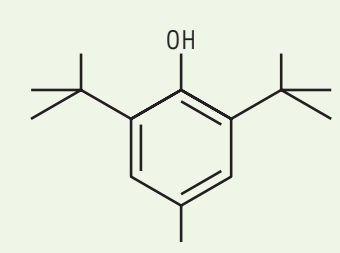

BHT

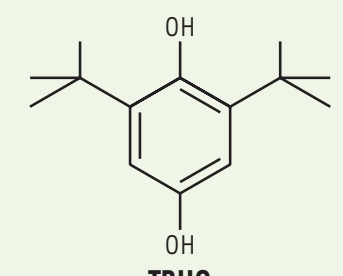

TBHP<smiles>COc1ccc(O)c(C(C)(C)C)c1</smiles>

BHA

\section{Évaluation de l'efficacité antioxydante d'un additif}

La mesure du potentiel antioxydant et le suivi des processus d'oxydation sont abordés globalement en déterminant des produits résultant de l'oxydation ou en évaluant l'aptitude à piéger des radicaux de modèles réactionnels. Le premier mode, plus ancien, nécessite une connaissance préalable des composés issus de l'oxydation. En effet ces méthodes recherchent certains groupements fonctionnels (aldéhydes, cétones, dicarbonylés...) dans les dérivés des constituants d'origine. Le second relie la quantité de radicaux piégés à celle d'antioxydant utilisé.

\section{Évaluation de produits résultant de l'oxydation}

L'efficacité des antioxydants (traduite en rapport d'inhibition, IR) correspond à :

$$
\operatorname{IR}(\%)=[(a-b) /(a-c)] \times 100
$$

$a, b, c$ sont les concentrations en dérivés oxydés respectivement, sans antioxydant et en présence de l'antioxydant à tester après incubation et sans antioxydant 
avant incubation. Le dosage des produits formés (en particulier des hydroperoxydes) est effectué par des techniques photométriques plus ou moins directes (Tableau I).

\section{Évaluation de l'aptitude}

du composé à piéger

des radicaux libres

La capacité du produit à piéger les radicaux libres et donc à ralentir ou inhiber, aussi bien les phases d'ini-

Spectrophotométrie directe d'hydroperoxydes insaturés [7, 8]

Dosage chimique des dérivés oxydés

- Évaluation des peroxydes $[7,9]$

- Dosage d'hydroperoxydes [11-13]

- Détermination du malonaldéhyde [14-16]

Examen de dérivés carbonylés sous forme de dinitrophénylhydrazones [9, 12, 19]

Évaluation de la dégradation du $\beta$-carotène en présence d'acide linoléique [17, 20-22]

Tableau I. Techniques d'évaluation des produits résultant de l'oxydation.

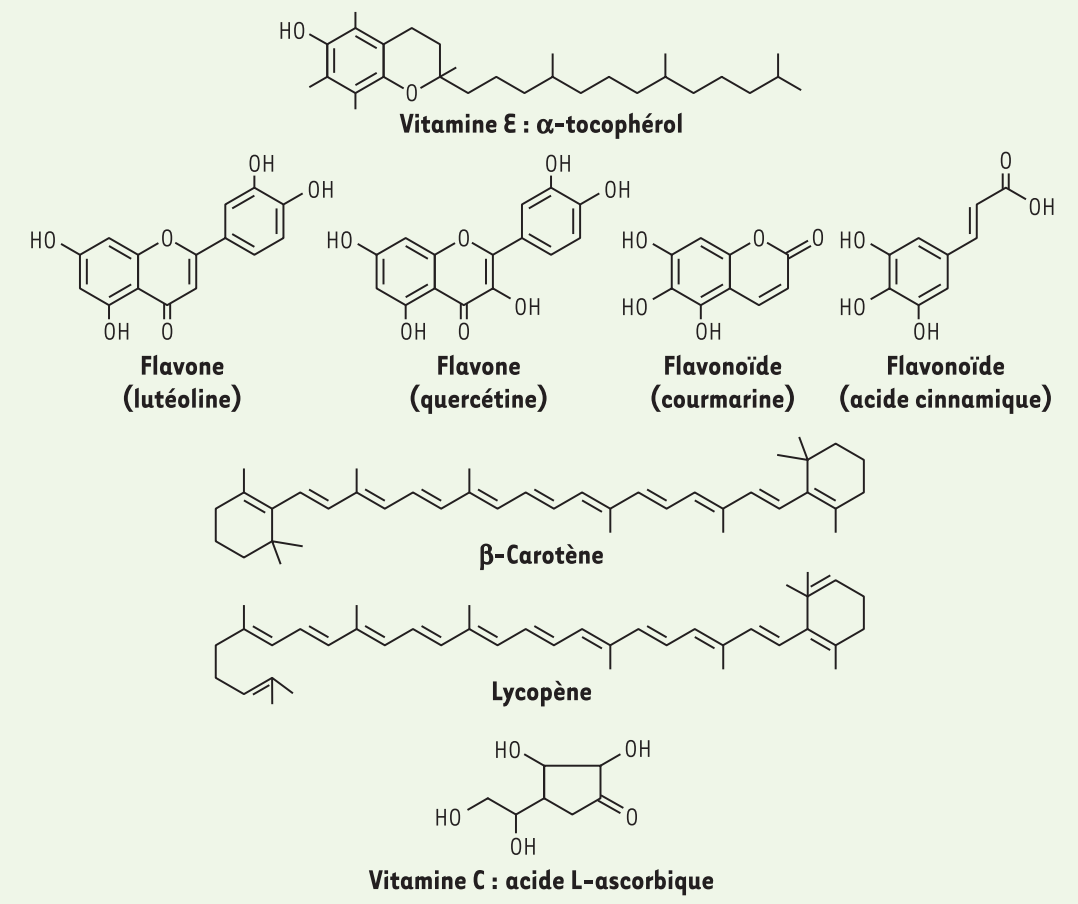

Figure 2. Principaux composés naturels (ou synthétisés) possédant des propriétés antioxydantes. La vitamine $\varepsilon$ est abondante dans les germes de blé, les légumes verts, les corps gras. Les flavones et flavonoïdes sont retrouvées dans les fruits, le vin, le thé. Les caroténoïdes sont présents dans les carottes, les fruits rouges et jaunes, les légumes verts ( $\beta$-carotène), les tomates (lycopène). La vitamine $C$ est abondante dans les agrumes, les fruits rouges, les pommes de terre primeur, les brocolis. tiation que de propagation, implique la création de radicaux. Dans la méthode TEAC (Trolox equivalent antioxidant capacity), l'activité antioxydante totale d'une molécule est déduite de sa capacité à inhiber le radical $A B T S^{\cdot+}$, obtenu à partir de l'ABTS (sel d'ammonium de l'acide 2,2'-azinobis-(3-éthylbenzothiazoline-6-sulfonique)) comparativement à un antioxydant de référence : le Trolox ${ }^{\circledR}$ (acide 6hydroxy-2, 5, 7,8-tétraméthylchroman-2-carboxylique), dont la structure moléculaire cyclique est similaire à celle de la vitamine $\varepsilon$. L'obtention du radical cation résulte $d u$ contact de I'ABTS avec une enzyme de peroxydation (peroxydase metmyoglobine [23] ou horseradish peroxidase) [24] en présence de $\mathrm{H}_{2} \mathrm{O}_{2}$ ou d'un oxydant (dioxyde de manganèse [25, 26] ou persulfate de potassium [27]). Le radical ABTS $^{+}$, en contact avec un donneur de $\mathrm{H}^{*}$ conduit à I'ABTS $^{+}$(Figure 3) et à la décoloration à $734 \mathrm{~nm}$ de la solution [28]. D'autres auteurs utilisent l'acide 2,2'azinobis-(3-éthylbenzothiazoline-6-sulfonique), ou ABTS ${ }^{-}$, à la place de son sel d'ammonium et analysent l'inhibition du radical ABTS *- , produit par un initiateur de radicaux thermolabiles, I'ABAP (2,2'-azobis-(2-amidinopropane) $\mathrm{HCl}$ ) [29]. La cinétique de réaction de l'antioxydant étudié doit être examinée préalablement pour déterminer la fin de réaction. La capacité antioxydante en équivalent Trolox ${ }^{\circledR}$ (TEAC) correspond à la concentration (mmole/l ou mg/l) de Trolox ${ }^{\circledR}$ ayant la même activité qu'une même concentration unitaire de substance à tester, jus de fruit par exemple [23]. La littérature fournit le TEAC de certains antioxydants, respectivement pour la vitamine $C$ et le $\beta$-carotène : $0,99 \mathrm{mM}$ [23] et 1,9 mM [26]. Un kit des Laboratoires Randox (Paris, France) permettant l'analyse en routine du statut antioxydant total du sérum humain est adaptable aux aliments.

D'autres méthodes sont également utilisées comme la méthode automatisée ORAC (oxygen radical absorbance capacity) [30-33], la méthode utilisant le radical stable DPPH (2,2-diphényl-1picrylhydrazyle) [20, 34-37], la mesure de l'aptitude à réduire $\mathrm{Fe}^{3+}$ en $\mathrm{Fe}^{2+}[38]$, et la méthode spectroscopique par résonance électronique (ESR) [38, 39]. 


\section{Conclusions}

Les principales méthodes d'évaluation du potentiel antioxydant d'un produit pur ou de mélanges ont été examinées et regroupées selon leurs principes. Elles sont fondées sur la détermination de produits résultant de l'oxydation ou, au contraire, mesurent l'efficacité d'une substance à piéger des radicaux, souvent en donnant une forme $\mathrm{H}^{\circ}$.

Parmi les méthodes d'évaluation, celles comparant l'aptitude du produit à celle du Trolox ${ }^{\circledR}$ (Ia méthode TEAC en particulier) sont intéressantes. En effet, elles mesurent directement la réactivité d'un composé donneur de $\mathrm{H}^{*}$, sans examen d'un produit de dégradation. L'examen au laboratoire de la méthode du TEAC a montré qu'elle était applicable dans différents solvants (diméthylsulfoxide, méthanol, éthanol ou tampon phosphate) quelle qu'en soit I'hydrophilie ou I'hydrophobie. La méthode automatisée ORAC diffère de celle du TEAC et du DPPH [40] en évaluant l'activité antioxydante de manière dynamique avec des

\section{SUMMARY}

Studies of several analytical methods for antioxidant potential evaluation in food

Oxidation control is necessary to manage the evolution of complex biological system, particularly in food whose degradation could have consequences on food security. After description of context and oxidation mechanisms, several analytical methods to evaluate the additive antioxidant potential are presented. This evaluation is performed either by quantification of products (in particular hydroperoxydes) using direct or indirect photometric techniques and chemical titration with suitable reactants or on the effectiveness to trap free radicals with modelized systems that can generate them. Methods based on the comparison of radical trapping ability between an additive and Trolox (particularly Trolox ${ }^{\circledR}$ equivalent antioxydant capacity, TEAC) can be applied to many products whatever the hydrophily or the hydrophobia of the medium. $\diamond$

radicaux actifs sur le plan pathologique.

Disposant de méthodes d'évaluations diversifiées il devient possible, d'une part, de suivre l'évolution des substances naturelles présentes dans les matières premières jouant un rôle d'antioxydant et éventuellement d'envisager un enrichissement pour atteindre l'objectif qualitatif souhaité et, d'autre part, de tester comme conservateurs différentes molécules employées pour d'autres usages (colorants alimentaires par exemple). Dans ces conditions «l'adjuvance antioxydative $\gg$, fruit d'une collaboration entre technologues et nutritionnistes, peut devenir beaucoup plus précise, tout en étant aussi efficace à moindre risque.

À défaut de disposer d'une électrode électrochimique accédant directement au niveau et à l'importance de la protection contre l'oxydation, les différentes méthodes présentées permettent, en fonction des milieux alimentaires étudiés, une évaluation optimale et ciblée du potentiel antioxydant. Elles sont complémentaires les unes des autres, sans que l'une puisse être établie en tant que référence fondamentale. $\diamond$

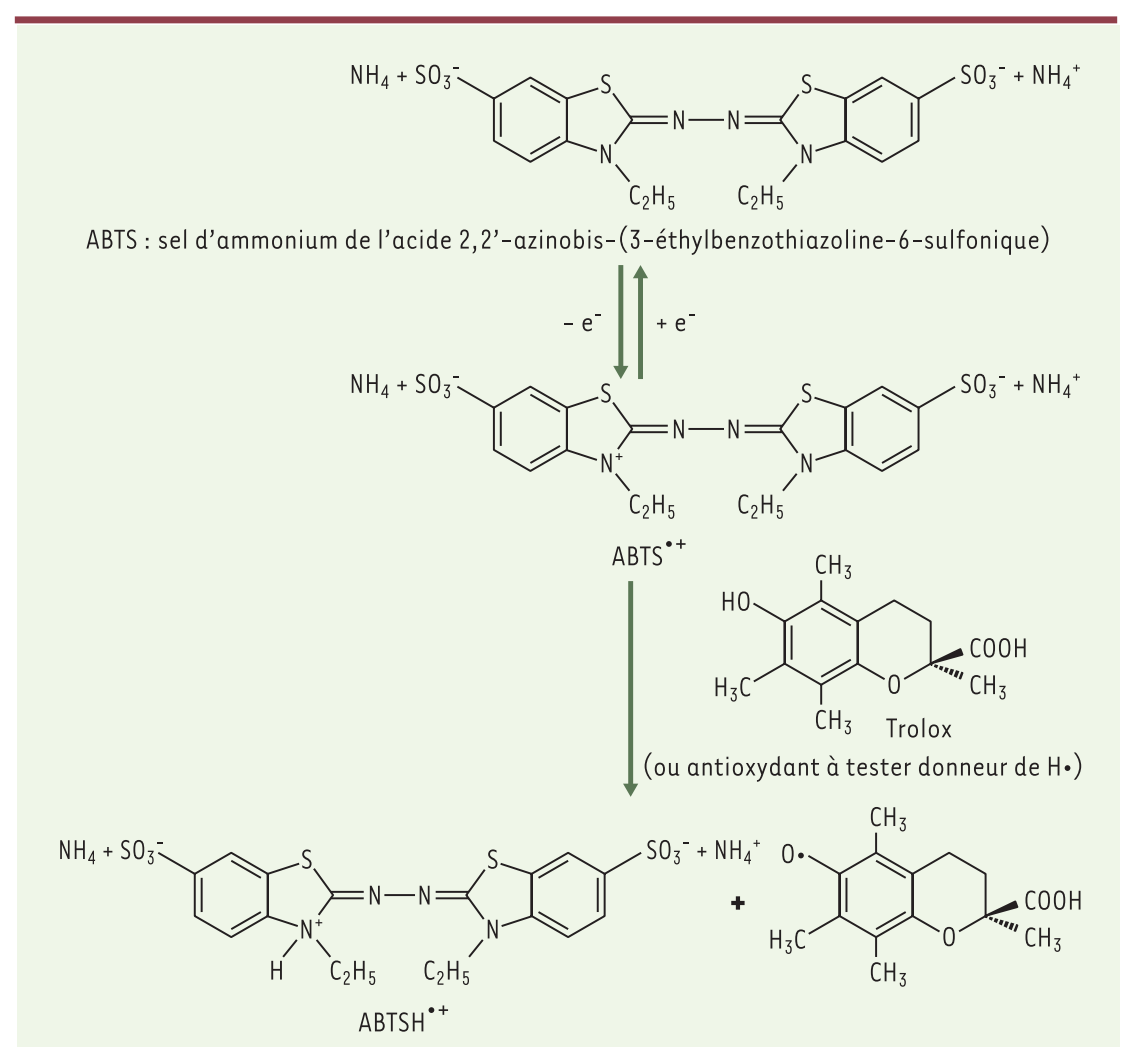

Figure 3. Formation et piégeage du radical $A B T S^{*}$ par un antioxydant donneur de $H^{*}$. Le radical ABTS $^{*+}$ (absorbant à $734 \mathrm{~nm}$ ) est formé par arrachement d'un électron e- à un atome d'azote de I'ABTS. En présence de Trolox (ou d'antioxydant donneur de $\mathrm{H}^{*}$ ), l'atome d'azote concerné piège un $\mathrm{H}^{*}$, conduisant à l'ABTS', ce qui entraîne la décoloration de la solution [28]. 


\section{RÉFÉRENCES}

1. Labuza TP. Sorption phenomena in foods : theoretical and practical aspects. In : Rha CK, ed. Theory determination and control of physical properties of food materials. Dordrecht : Reidel D, 1975 : 197-219.

2. Chaveron H. Molécules toxiques. Molécules formées au cours des réactions d'oxydation. In : Chaveron $\mathrm{H}$, ed. Introduction à la toxicologie nutritionnelle. Londres : Tec Et Doc, 1999 : 175-83.

3. Moll M, Moll N. Les autres additifs. Les antioxygènes. In : Moll M, Moll N eds. Additifs alimentaires et auxiliaires technologiques, $2^{\mathrm{e}}$ ed, série Agro-Alimentaire. Paris : Dunod, 1998 : 89-99.

4. Penfield MP, Campbell AM. Fats and their lipids constituents. Chemical reactions of lipids. In : Penfield MP, Campbell AM. eds. Experimental food science, $3^{e}$ ed. San Diego : Academic Press, 1990 : 340-7.

5. Gordon MH. The mechanism of antioxidant action in vitro. In : Hudson BJF, ed. Food antioxydants. Amsterdam : Elsevier, 1990 : 1-18.

6. Moure A, Cruz JM, Franco D, et al. Natural antioxidants from residual sources. Food Chem $2001 ; 72: 45-171$.

7. Krings U, El-Saharty YS, El-Zeany BA, et al. Antioxidant activity of some extracts from roasted wheat germ. Food Chem $2000 ; 71$ : 91-5.

8. Pryor WA, Cornicelli JA, Devall LJ, et al. A rapid screening test to determine the antioxidants potencies of natural and synthetic antioxydants. J Org Chem $1993 ; 58: 3521-32$.

9. McDonald RE, Hultin HO. Some characteristics of the enzymic lipid peroxidation system in the microsomal fraction of flounder skeletal muscle. J Food Sci 1987 ; 52 : 15-27.

10. Mitsuda H, Yasumoto K, Iwami K. Antioxidative action of indole compounds during the autoxidation of linoleic acid. Eiyo Shokuryou $1966 ; 19: 210-4$

11. Yen GC, Duh PD, Chuang DY. Antioxidant activity of anthraquinones and anthrone. Food Chem $2000 ; 70: 437-41$.

12. Osawa T, Namiki M. A novel type of antioxidant isolated from leaf wax of eucalyptus leaves. Agric Biol Chem 1981; 45 : 735-9.

13. Jayaprakasha GK, Singh RP, Sakariah KK. Antioxidant activity of grape seed (Vitis vinifera) extracts on peroxidation models in vitro. Food Chem $2001 ; 73: 285-90$.

14. Siu GM, Draper HH. A survey of the malonaldehyde content of retail meats and fish. J Food Sci 1978 ; 43 : 1147-9.

15. Mihara M, Uchiyama M. Determination of malonaldehyde precursor in tissues by thiobarbituric acid test. Anal Biochem 1978; $86: 271-8$.

16. Fernandez J, Perez-Alvarez JA, Fernandez-Lopez JA. Thiobarbituric acid test for monitoring lipid oxidation in meat. Food Chem 1997 ; $59: 345-53$.

17. Mansour $\varepsilon H$, Khalil AH. Evaluation of antioxidant activity of some plant extracts and their application to ground beef patties. Food Chem 2000 ; $69: 135-41$.

18. McDonald S, Prenzler PD, Antolovich M, Robards K. Phenolic content and antioxidant activity of olive extracts. Food Chem $2001 ; 73: 73-84$

19. Kumazawa H, Oyama T. Estimation of total carbonyl content in oxidized oil by 2,4-dinitrophenylhydrazine. Yukagaku 1965; 14:15-9.

20. Lu Y, Foo Ly. Antioxidant and radical scavenging activities of polyphenols from apple pomace. Food Chem $2000 ; 68: 81-5$.

21. Terao J. Antioxidant activity of beta-carotene-related carotenoids in solution. Lipids $1989 ; 24: 659-61$.

22. Cuvelier ME, Berset C, Richard H. Use of a new test for determining comparative antioxidative activity of butylated hydroxyanisole, butylated hydroxytoluene, alpha- and gamma-tocopherols and extracts from rosemary and sage. Sciences des Aliments $1990 ; 10: 797-806$

23. Miller NJ, Rice-Evans CA. The relative contributions of ascorbic acid and phenolic antioxidants to the total antioxidant activity of orange and apple fruit juices and blackcurrant drink. Food Chem 1997 ; 60 : 331-7.

24. Arnao MB, Cano A, Acosta M. The hydrophilic and lipophilic contribution to total antioxidant activity. Food Chem $2001 ; 73$ : 239-44.

25. Benavente-Garcia 0, Castillo J, Lorente J, et al. Antioxidant activity of phenolics extracted from olea europaea L leaves. Food Chem 2000 $68: 457-62$.

26. Miller NJ, Sampson J, Candeias LP, et al. Antioxidant activities of carotenes and xanthophylls. FEBS Lett $1996 ; 384: 240-2$.

27. Re R, Pellegrini N, Proteggente A, et al. Antioxidant activity applying an improved ABTS radical cation decolorization assay. Free Radic Biol Med $1999 ; 26: 1231-7$
28. Lien $\varepsilon$ J, Ren S, Bui HH, Wang R. Quantitative structure-activity relationship analysis of phenolic antioxidants. Free Radic Biol Med $1999 ; 26: 285-94$.

29. Van Den Berg R, Haenen GR, Van Den Berg H, et al. The predictive value of the antioxidant capacity of structurally related flavonoids using the trolox equivalent antioxidant capacity (TEAC) assay. Food Chem 2000 ; $70: 391-5$.

30. Cao G, Verdon CP, Wu AH, et al. Automated assay of oxygen radical absorbance capacity with the COBAS FARA II. Clin Chem 1995 ; 41 : 1738-44.

31. Cao G, Sofic $\varepsilon$, Prior RL. Anioxidant and prooxidant behavior of flavonoids : structure-activity relationships. Free Radic Biol Med 1997 ; 22 : 749-60.

32. Cao G, Sofic $\varepsilon$, Prior RL. Antioxidant capacity of tea and common vegetables. J Agric Food Chem 1996 ; 44 : 3426-31.

33. Wang H, Cao G, Prior RL. Total antioxydant capacity of fruits. J Agric Food Chem 1996 ; 44 : 701-5.

34. Saija A, Trombetta D, Tomaino A, et al. In vitro evaluation of the antioxidant activity and biomembrane interaction of the plant phenols oleuropein and hydroxytyrosol. Int J Pharm 1998 ; 166 : 123-33.

35. Sreejayan N, Rao MN. Free radical scavenging activity of curcuminoids. Arzneimittelforschung $1996 ; 46: 169-71$.

36. Brand-Williams W, Cuvelier ME, Berset C. Use of a free radical method to evaluate antioxidant activity. Lebensm-Wiss U-Technol 1995 ; $28: 25-30$.

37. Hatano T, Kagawa H, Yasuhara T, Okuda T. Two new flavonoids and other constituents in Licorice roots ; their relative astringency and radical sacvenging effects. Chem Pharm Bull $1998 ; 36: 2090-7$.

38. PT, White TAC, McPhail DB, Duthie GG. The relative contributions of vitamin $C$, carotenoids and phenolics to the antioxidant potentiel of fruit juices. Food Chem $2000 ; 68: 471-4$.

39. Gardner PT, McPhail DB, Crozier A, Duthie GG. Electron spin resonance (ERS) spectroscopic assessment of the contribution of quercetin and other flavonols to the antioxidant capacity of red wines. J Sci Food Agric 1999 ; 79 : 1011-4.

40. Prior RL, Cao G. In vitro total antioxidant capacity : comparison of different analytical methods. Free Radic Biol Med 1999; 27 : 1173-81.

\section{TIRÉS À PART}

\section{F. Marc}

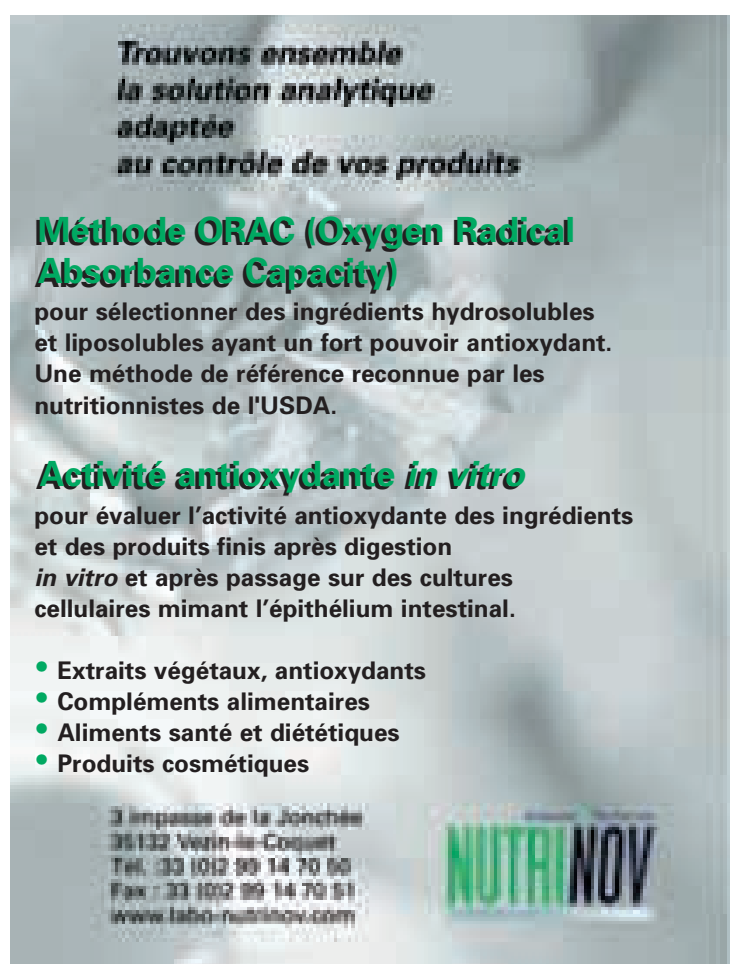

